\title{
Fault-Tolerant Quantum Computation with Higher-Dimensional Systems
}

\author{
Daniel Gottesmant \\ T-6 Group, Los Alamos National Laboratory
}

\begin{abstract}
Instead of a quantum computer where the fundamental units are 2-dimensional qubits, we can consider a quantum computer made up of $d$-dimensional systems. There is a straightforward generalization of the class of stabilizer codes to $d$-dimensional systems, and I will discuss the theory of fault-tolerant computation using such codes. I prove that universal fault-tolerant computation is possible with any higher-dimensional stabilizer code for prime $d$.
\end{abstract}

\section{Introduction}

Quantum computation and quantum communications have the potential to accomplish many things that would be difficult or impossible using just classical computers and communications. However, quantum data is very vulnerable to decoherence and to errors. It is likely that some form of quantum error correction will be needed to perform anything beyond the simplest computations with a quantum computer. Quantum error-correcting codes [1, 2, 3, 4, 5] provide one of the tools necessary. Such a code can protect quantum data against errors occuring during transmission or storage of the data. However, to have a reliable quantum computer, we also need for the computation to be performed in a fault-tolerant manner [6]. Fault-tolerant quantum computation requires a protocol that not only maps states of a quantum code to other states of a quantum code, but prevents errors from propagating out of control.

A large group of useful codes was introduced in [3] and [4]. These codes are essentially the quantum equivalent of classical linear codes, in that they can be easily described and encoded, and that it is easy to measure the error syndrome (as in the classical case, it may be difficult to compute the actual error from the error syndrome). The primary complication involved in quantum error correction is that it is not only necessary to correct bit flip errors

$$
X=\left(\begin{array}{ll}
0 & 1 \\
1 & 0
\end{array}\right)
$$

but also phase errors

$$
Z=\left(\begin{array}{cc}
1 & 0 \\
0 & -1
\end{array}\right)
$$

\footnotetext{
* gottesma@t6-serv.lanl.gov
} 
Consequently, it turns out to be useful to look at the group $\mathcal{P}$ generated by tensor products of these operators. $\mathcal{P}$ is called the Pauli group or the extraspecial group. Stabilizer codes are those codes where the valid codewords are all eigenstates of $n-k$ operators in $\mathcal{P}$. These $n-k$ operators generate a $2^{n-k}$ element Abelian group, called the stabilizer $S$ of the code. The set of valid codewords forms a $2^{k}$-dimensional subspace of the full $n$-qubit Hilbert space, the coding space of the code. The stabilizer is analogous to the parity check matrix of a classical linear code. In fact, the set of classical linear binary codes is exactly the set of stabilizer codes where everything in the stabilizer is a tensor product of $Z$ operators.

Stabilizer codes are easy to work with because of the structure of the Pauli group.

$$
X Z=-Z X,
$$

so any two operators in $\mathcal{P}$ either commute or they anticommute. If an operator $E$ anticommutes with an operator $G$ in the stabilizer $S$ of a code, then $E|\psi\rangle$ will have eigenvalue -1 for $G$ instead of +1 . Therefore, by measuring the eigenvalues of the $n-k$ generators of $S$, we can easily measure the error syndrome, and if the code is suitably chosen, identify the error.

Even if we restrict our attention to stabilizer codes, it is far from obvious that we can perform fault-tolerant computation. First of all, we must find some operators that map the coding space of the code into itself. Secondly, many of these operators will cause errors to spread from one qubit in a block to a different qubit in the same block of the code. Therefore, a single error could rapidly grow to become many errors within a block, exceeding the code's capability to correct them. In order to avoid this, we will further restrict attention to transversal operations, that is, operations which only interact qubits from one block with corresponding qubits in other blocks. This means that an error occuring in qubit number 3 in one block might spread to qubit number 3 in another block, but it will never spread back to qubit 2 in the first block. Each block can easily correct a single error, so this situation causes no problems. Shor was the first to demonstrate a universal set of fault-tolerant gates [6]. However, his construction only worked for a small class of codes. In [7], I was able to show that Shor's construction could be generalized to any qubit stabilizer code. The proof made extensive use of the group of unitary operators that leave the group $\mathcal{P}$ invariant under conjugation. This group is known as the Clifford group.

In the classical theory of error-correcting codes, it is often helpful to go beyond bits and work with higher-dimensional systems. The same may be true for quantum error correction. Instead of a system made up of 2-dimensional qubits, we can work with a system composed of $d$-dimensional qudits. It turns out that there is a natural generalization of stabilizer codes to higher-dimensional systems 8, 9. 10]. In Sec. 2, I will present this generalization. Then I will procede to generalize the arguments of 77 to show that universal fault-tolerant computation is also possible with any of these codes, at least in the case where $d$ is prime. Though I will largely focus on prime $d$, I will also say a little bit about the general case along the way. Assume that $d$ is prime unless it is otherwise specified. 
The construction of a universal set of gates used in [7] consisted of a number of steps:

1. The full Clifford group can be constructed given the controlled-NOT, operators in the Pauli group, and measurement of operators in the Pauli group.

2. For any stabilizer code, we can perform encoded versions of all operators in the Pauli group and can measure operators in the Pauli group.

3. We can perform a CNOT between corresponding encoded qubits in different blocks of the code.

4. We can swap individual encoded qubits from one block of the code to an empty block and vice-versa.

5. We can move an encoded qubit in an otherwise empty block to whatever position in the block we desire. At this point, we have established that we can perform a CNOT between any pair of encoded qubits in the same or different blocks, and therefore can perform the full Clifford group.

6. Given the full Clifford group, we can perform an additional gate outside the Clifford group, such as the Toffoli gate or the $\pi / 8$ rotation. This completes the universal set of gates.

Each of the steps in this construction has an analog for higher-dimensional systems. However, in this paper, I will present a simplified construction that combines steps 3 through 5 . This construction gives a direct way to perform the generalization of the CNOT between any pair of encoded qudits, whether they are in the same or different blocks and whether they are in corresponding or different positions within their blocks. This construction can also be used to simplify the proof for systems with $d=2$, and will likely reduce the required overhead for fault-tolerant computation using codes with many qudits per block.

\section{Higher Dimensional Generalization of the Pauli Group and Other Structures}

The Pauli group has a natural generalization to higher-dimensional systems. Instead of generating it from the two-dimensional $X$ and $Z$, we instead generate $\mathcal{P}$ from tensor products of $X_{d}$ and $Z_{d}$, where $X_{d}|j\rangle=|j+1\rangle$ and $Z_{d}|j\rangle=\omega^{j}|j\rangle$, where $\omega$ is a primitive $d$-th root of unity. $X_{d}$ and $Z_{d}$ satisfy the relation

$$
X_{d} Z_{d}=\omega^{-1} Z_{d} X_{d} .
$$

Both $X_{d}$ and $Z_{d}$ have order $d$. The elements of the single-qudit Pauli group have the form $\omega^{a} X_{d}^{r} Z_{d}^{s}$, where $0 \leq r, s<d$, and

$$
\left(X_{d}^{r} Z_{d}^{s}\right)\left(X_{d}^{t} Z_{d}^{u}\right)=\omega^{s t-r u}\left(X_{d}^{t} Z_{d}^{u}\right)\left(X_{d}^{r} Z_{d}^{s}\right)
$$

2 The group presented in this section is not the only generalization of the Pauli group, although it is probably the simplest. See 8 for a more extensive discussion of this issue. 
$\mathcal{P}$ for $n$ qubits will contain $d^{2 n}$ elements, plus an additional factor of $d$ for overall phase. The elements of $\mathcal{P}$ have eigenvalues $\omega^{r}$ for $r=0, \ldots, d-1$ From now on, I will suppress the subscript $d$, and all operations should be taken to be over qudits instead of qubits.

The $d$-dimensional generalization of the stabilizer $S$ of a code is again just an Abelian subgroup of $\mathcal{P}$. The coding space is composed of those states that are fixed by all elements of $S$ (when $d$ is even, this actually imposes an additional constraint on the overall phase of elements of $S$ ). If the stabilizer on $n$ qudits has $n-k$ generators, then $S$ will have $d^{n-k}$ elements and the coding space will consist of $k$ qudits. Note that this last fact need no longer be true when $d$ is not prime, and this is the main source of complications in that case. It is unclear exactly how to deal with a code that does not encode an integral number of qudits. If we stick to codes for which all the generators of the stabilizer have order $d$, the rest of the proof will hold, modulo a question about gates necessary to generate the Clifford group.

If an operator $E$ and $M \in S$ satisfy

$$
E M=\omega^{a} M E,
$$

then $E|\psi\rangle$ will have eigenvalue $\omega^{a}$ for $M$ instead of eigenvalue +1 , so we can detect that error $E$ has occurred by measuring the eigenvalue of $M$.

We can see the structure of the coding space by extending the generators of $S$ to a complete independent set of commuting operators. When $d$ is not prime, we also require these operators to have order $d$. Such a set will have cardinality $n$, so we can do this by choosing $k$ additional operators $\bar{Z}_{1}, \ldots, \bar{Z}_{k}$. These operators have the interpretation of the encoded $Z$ operators for the $k$ encoded qudits. We can then choose $k$ more operators $\bar{X}_{1}, \ldots, \bar{X}_{k}$ which satisfy the relations

$$
\begin{aligned}
& \bar{X}_{i} \bar{Z}_{j}=\bar{Z}_{j} \bar{X}_{i}(i \neq j) \\
& \bar{X}_{i} \bar{Z}_{i}=\omega^{-1} \bar{Z}_{i} \bar{X}_{i} \\
& \bar{X}_{i} M=M \bar{X}_{i}(\forall M \in \mathcal{P}) .
\end{aligned}
$$

The operators $\bar{X}_{i}$ then act as the encoded $X$ operators for the $k$ encoded qudits. The generators of $S$ along with $\bar{Z}_{1}, \ldots, \bar{Z}_{k}$ and $\bar{X}_{1}, \ldots, \bar{X}_{k}$ then generate the group of all Pauli group operators that commute with $S$. As in the twodimensional case, the operators that commute with $S$ but are not themselves in $S$ are precisely the operators that cannot be detected by the quantum code. They therefore perform encoded operations on the data.

The Clifford group is the set of operators that leave $\mathcal{P}$ invariant under conjugation. That is, it is the normalizer $N(\mathcal{P})$ of $\mathcal{P}$ in the unitary group $U\left(d^{n}\right)$. The Clifford group is important for fault-tolerant computation because if we perform an operator $U$ on the Hilbert space, the operator $U N U^{\dagger}$ has the same relationships to states after the transformation as the operator $N$ did before the transformation. Therefore, instead of considering transformations of the states

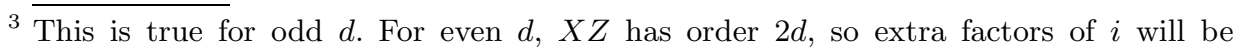
necessary, as in the $d=2$ case. This aspect is actually simpler for odd $d$ than for $d=2$. 
$|\psi\rangle \rightarrow U|\psi\rangle$, we can consider transformations of the operators $N \rightarrow U N U^{\dagger}$. When $U$ is in the Clifford group and $N$ is in the Pauli group, then $U N U^{\dagger}$ will also be in the Pauli group. Therefore, we can uniquely describe elements of $N(\mathcal{P})$ by the permutation they induce on $\mathcal{P}$. The permutation must preserve the group structure of $\mathcal{P}$, but is otherwise arbitrary.

In the two-dimensional group, $N(\mathcal{P})$ was generated by two single-qubit operators $R$ (the Hadamard transform $|j\rangle \rightarrow|0\rangle+(-1)^{j}|1\rangle$ ) and $P$ (the phase gate $\left.|j\rangle \rightarrow i^{j}|j\rangle\right)$, and the two-qubit operator CNOT $(|i\rangle|j\rangle \rightarrow|i\rangle|(i+j) \bmod 2\rangle)$. In $d$ dimensions, $R$ generalizes to the $d$-dimensional discrete Fourier transform

$$
|j\rangle \rightarrow \sum_{s=0}^{d} \omega^{j s}|s\rangle,
$$

$P$ generalizes to the $d$-dimensional phase gate

$$
|j\rangle \rightarrow \omega^{j(j-1) / 2}|j\rangle,
$$

and CNOT generalizes to the SUM gate

$$
|i\rangle|j\rangle \rightarrow|i\rangle|(i+j) \bmod d\rangle
$$

We can describe these operators by their induced transformations on the Pauli group. $R$ maps

$$
\begin{gathered}
X \rightarrow Z, \\
Z \rightarrow X^{-1} .
\end{gathered}
$$

$P$ maps

$$
\begin{gathered}
X \rightarrow X Z \\
Z \rightarrow Z
\end{gathered}
$$

SUM maps

$$
\begin{aligned}
& X \otimes I \rightarrow X \otimes X, \\
& I \otimes X \rightarrow I \otimes X, \\
& Z \otimes I \rightarrow Z \otimes I, \\
& I \otimes Z \rightarrow Z^{-1} \otimes Z .
\end{aligned}
$$

However, it is not clear that these three gates generate the Clifford group. We may also need the $S$ gate

$$
\begin{gathered}
X \rightarrow X^{a}, \\
Z \rightarrow Z^{b},
\end{gathered}
$$

for all pairs $(a, b)$, where $a b \equiv 1 \bmod d$. On kets, this gate acts as $|j\rangle \rightarrow|a j\rangle$. In fact, a single pair $(a, b)$ is sufficient, as long as $a$ generates the multiplicative group $\mathbb{Z}_{d}^{*}$. I will not give a detailed proof that these gates generate the Clifford 
group, but using the $P, R$, and $S$ gates, we can get the full one-qudit Clifford group. Then a construction similar to that used in [7] will give the full $n$-qudit Clifford group. The structure is somewhat more complicated when $d$ is not prime, and I have not verified that these gates are sufficient for the nonprime case.

Note that we can fault-tolerantly measure any operator in $\mathcal{P}$ that is the tensor product of $Z$ operators by performing transversal SUM gates from the qudits to be measured to an appropriate ancilla. Since we are interested in eigenvalues with possible values $\omega^{j}$ for $j=0, \ldots, d-1$, the appropriate ancilla state is the superposition of all states where the sum of the qudits is $0 \bmod d$. That way, no information beyond the eigenvalue of the measured operator will be conveyed. We can construct this state by Fourier transforming the state $\sum_{j=0}^{d-1}|j j \cdots j\rangle$. Following DiVincenzo and Shor [11, we can measure any operator in $\mathcal{P}$ by performing a transversal Clifford group operation $C$ that takes the operator to the tensor product of $Z$ 's, performing the measurement, and applying $C^{-1}$.

For any stabilizer code, the elements of the $k$-qudit encoded Pauli group are also elements of the $n$-qudit unencoded Pauli group, as are the generators of the stabilizer. Since we can perform and measure an arbitrary element of the unencoded Pauli group, we have shown that for a stabilizer code over $d$ dimensions, we can apply encoded versions of $X$ and $Z$ for all encoded qudits, measure the generators of the stabilizer (and therefore perform fault-tolerant error correction), and measure all members of the encoded Pauli group faulttolerantly. This provides step 2 of the proof.

\section{Measurements and Stabilizers}

In 四, it proved very helpful in a number of places to understand how the stabilizer of a state or subspace changed under measurements. The procedure for qubits generalizes easily to higher dimensions.

First, recall that there is more than one way to choose generators for a given stabilizer. Any maximal set of independent operators in the group will suffice. In particular, any generator $M$ can be replaced by $N M$ for any $N \neq M$. Similarly, the encoded $\bar{X}$ and $\bar{Z}$ operators are only defined up to multiplication by elements of $S$. If we wish to measure an operator $A \in \mathcal{P}$, then the first step is to put the stabilizer and $\bar{X}$ and $\bar{Z}$ operators in a form so that all the $\bar{X}$ and $\bar{Z}$ operators commute with $A$ and all but one of the generators of $S$ commutes with $A$. We can do this because if $M \in S$ does not commute with $A$, then $M^{a} N$ will commute with $A$ for some $a$ for any $N$ that commutes with $M$ (as do all $\bar{X}$ 's, $\bar{Z}$ 's, and generators of $S$ ). We will not need to consider the case where $A$ commutes with everything in the stabilizer.

This is a useful form for the stabilizer because any operator that commutes with $A$ is not disturbed by the measurement of $A$. Therefore, we only need to change $M$ when $A$ is measured. Since $A \in \mathcal{P}$, the possible measurement results are $\omega^{a}$ for $a=0, \ldots, d-1$. These result $\omega^{a}$ corresponds to applying the projection operator

$$
P_{a}=\left(I+\omega^{-a} A+\omega^{-2 a} A^{2}+\cdots+\omega^{-(d-1) a} A^{d-1}\right) / d
$$


to the state. Assume now that

$$
M A=\omega A M
$$

(note that when $d$ is prime, this will always be true for some power of $M$ ). Then

$$
M P_{a} M^{\dagger}=\frac{1}{d} \sum_{j=0}^{d-1} \omega^{-j a} M A^{j} M^{\dagger}=\frac{1}{d} \sum_{j=0}^{d-1} \omega^{-j(a-1)} A^{j}=P_{a-1} .
$$

Thus, if we measure $A$ and get the result $a$, by applying $M^{a}$ we can produce the same state we would have gotten if we had gotten the result 0 . I will assume below that any measurement is followed by such a correction. Once this correction is performed, the new state is a +1 eigenvector of $A$, so $A$ should be added to the stabilizer. It is not an eigenvector of $M$, so $M$ is removed from the stabilizer. All of the other generators (which have been put in a form where they commute with $A$ ) are unchanged.

\section{Gates Derived from SUM}

Suppose we have the ability to perform the SUM gate between any pair of qudits in our computer, as well as the ability to perform the Pauli group and to measure operators in the Pauli group. I will now show that we can apply the full Clifford group to the computer.

Suppose we consider a single unknown qudit and prepare a second ancilla qudit in the state $|0\rangle$. This two-qudit system can be described by the stabilizer $I \otimes Z$. The logical Pauli group is generated by $\bar{X}=X \otimes I$ and $\bar{Z}=Z \otimes I$. Now perform a SUM gate from the first qudit to the second qudit. The stabilizer is $Z^{-1} \otimes Z, \bar{X}=X \otimes X$, and $\bar{Z}=Z \otimes I$.

Suppose we were now to measure the operator $A=I \otimes X Z$. Then $M=$ $Z^{-1} \otimes Z \in S$ and $M A=\omega A M$. Therefore, this measurement results in the stabilizer $I \otimes X Z$, and $\bar{X}=X Z^{-1} \otimes X Z$ and $\bar{Z}=Z \otimes I$. We can discard the second qudit, and the effective transformation on the first qudit is

$$
\begin{aligned}
& X \rightarrow X Z^{-1}, \\
& Z \rightarrow Z .
\end{aligned}
$$

This is the gate $P^{-1} \cdot d-1$ iterations of it will produce the $P$ gate.

Alternatively, we could have prepared the ancilla qudit so that the stabilizer of the system began as $I \otimes X$, then performed the SUM gate from the second qudit to the first. The stabilizer would then be $X \otimes X$, and $\bar{X}=X \otimes I$ and $\bar{Z}=Z \otimes Z^{-1}$. Then we measure $A=I \otimes X Z^{-1}$ and choose $M=X \otimes X$ so that $M A=\omega A M$. The final stabilizer is $I \otimes X Z^{-1}$, so we discard the second qudit, leaving

$$
\begin{gathered}
X \rightarrow X \\
Z \rightarrow X Z
\end{gathered}
$$


Call this gate $Q$. Then $R^{-1}=X Q P^{-1} Q$, and $R=R^{-3}$.

Now suppose we again prepare the ancilla in the +1 eigenstate of $X$, but now perform $s$ SUM gates from the second qudit to the first instead of one. The stabilizer is $X^{s} \otimes X, \bar{X}=X \otimes I$, and $\bar{Z}=Z \otimes Z^{-s}$. This time we measure $A=Z \otimes I$. This results in stabilizer $Z \otimes I$,

$$
\bar{X}=(X \otimes I)\left(X^{s} \otimes X\right)^{-s^{-1}}=I \otimes X^{-s^{-1}},
$$

and $\bar{Z}=Z \otimes Z^{-s}$. Therefore, discarding the first qudit leaves the transformation

$$
\begin{aligned}
X & \rightarrow X^{-s^{-1}}, \\
Z & \rightarrow Z^{-s} .
\end{aligned}
$$

By choosing an appropriate $s$, we can therefore perform an arbitrary $S$ gate. Note that in this case, the data ends up in what was originally the ancilla qudit.

I have shown how to produce the $P, R$, and $S$ gates from the SUM gate. Therefore, given the SUM gate, we can produce the full Clifford group. This completes step 1 of the proof.

\section{Producing the SUM gate for any stabilizer code}

To see how to construct the SUM gate between any pair of encoded qudits, first consider two unencoded qudits. Introduce a third qudit in the state $|0\rangle$. The stabilizer at this point is $I \otimes I \otimes Z$. Assume we can do Pauli group measurements, even entangled ones, and perform operators in the Pauli group. Let us first measure the operator $I \otimes X \otimes X^{-1}$. This becomes the stabilizer. The logical Pauli group generators are

$$
\begin{aligned}
& \bar{X}_{1}=X \otimes I \otimes I, \\
& \bar{X}_{2}=I \otimes X \otimes I, \\
& \bar{Z}_{1}=Z \otimes I \otimes I, \\
& \bar{Z}_{2}=I \otimes Z \otimes Z .
\end{aligned}
$$

Now measure $Z \otimes I \otimes Z$. It becomes the new stabilizer, and

$$
\begin{aligned}
& \bar{X}_{1}=X \otimes X \otimes X^{-1}, \\
& \bar{X}_{2}=I \otimes X \otimes I, \\
& \bar{Z}_{1}=Z \otimes I \otimes I, \\
& \bar{Z}_{2}=I \otimes Z \otimes Z .
\end{aligned}
$$

Finally, measure $I \otimes I \otimes X$ and discard the last qudit. This leaves us with

$$
\begin{aligned}
& \bar{X}_{1}=X \otimes X, \\
& \bar{X}_{2}=I \otimes X, \\
& \bar{Z}_{1}=Z \otimes I, \\
& \bar{Z}_{2}=Z^{-1} \otimes Z .
\end{aligned}
$$


This we recognize as the transformation induced by the SUM gate, so this series of entangled measurements has performed the SUM gate between these two qudits.

Now, to apply this to a quantum code, we just need to be able to measure entangled logical Pauli group operators between any pair of encoded qudits. If the qudits are in the same block, this is straightforward. For instance, if they are in slots $i$ and $j$, the encoded version of $X \otimes X$ is just $\bar{X}_{i} \bar{X}_{j}$. This is in the Pauli group too, so we know how to measure it.

If the qudits are in different blocks, it is not much harder. Instead of using an $a$-qudit ancilla state, we use an $(a+b)$-qudit ancilla state (where $a$ and $b$ are the weights of the operators $\bar{X}_{i}$ and $\bar{X}_{j}$ ), which is again in the superposition of all states whose registers sum to $0 \bmod d$. The operator we wish to measure is $\bar{X}_{i} \otimes \bar{X}_{j}$, which is in the Pauli group. By performing the appropriate transversal Clifford group operation, we rotate this to be the tensor product of $Z$ 's and perform SUM gates from the appropriate qudits to the corresponding ancilla qudits, then perform the inverse Clifford group operator to restore the state to its original form. Then we measure the $a+b$ ancilla qudits, and this tells us the eigenvalue of the measured operator. We use an $(a+b)$-qudit ancilla instead of an $a$-qudit ancilla plus a $b$-qudit ancilla because we do not wish to be able to find the eigenvalues of $\bar{X}_{i} \otimes I$ and $I \otimes \bar{X}_{j}$ separately, only their product.

Therefore, given an encoded ancilla qudit which is initialized to $|0\rangle$, by performing the encoded version of the above entangled measurements, we can perform a SUM gate between any pair of encoded qudits anywhere in the computer. Note that the ancilla qudit can itself be anywhere in the computer; it need not be in the same block as either data qudit, or in the corresponding place in a different block.

Given the SUM gate and the results of the previous section, we can perform the full Clifford group on the encoded data for any stabilizer code. This completes the proof up to step 5. This part of the proof is a significant improvement on the method used in [7]. In that paper, it was necessary to introduce full ancilla blocks to perform a CNOT. Here, we need only a single logical ancilla qudit. In the case where a block may encode many qudits, this can be a major improvement. The price is that we must potentially perform entangled measurements on more than one block. This means we will have to use larger ancilla states for the measurement; this results in a greater potential for error, so we will have to repeat the measurement more times, and perhaps perform error correction a bit more often. However, in many situations, the total number of physical gates we need will decrease.

Note that this procedure works just as well if the two logical qudits involved in the SUM are in blocks made up of different numbers of physical qudits. This means we can interact qudits encoded with different sorts of codes faulttolerantly, or change the encoding of a single qudit without losing the protection against errors at any time. 


\section{Completing the Universal Set of Gates}

The set of universal gates can be completed by adding the higher-dimensional analog of the Toffoli gate 12]

$$
|a\rangle|b\rangle|c\rangle \rightarrow|a\rangle|b\rangle|c+a b\rangle
$$

It turns out that a generalization of Shor's fault-tolerant construction of the Toffoli gate [6] will work here.

Suppose we prepare a three-qudit ancilla in the +1 eigenstate of the three operators

$$
\begin{aligned}
& M_{1}=(X \otimes I \otimes I) \operatorname{SUM}(2 \rightarrow 3), \\
& M_{2}=(I \otimes X \otimes I) \operatorname{SUM}(1 \rightarrow 3), \\
& M_{3}=(I \otimes I \otimes Z) \operatorname{PHASE}(1,2)^{-1} .
\end{aligned}
$$

$\operatorname{SUM}(i \rightarrow j)$ is a SUM gate performed with the $i$ th qudit as control and the $j$ th qudit as target. $\operatorname{PHASE}(i, j)$ is the PHASE gate

$$
\text { PHASE }|a\rangle|b\rangle=\omega^{a b}|a\rangle|b\rangle
$$

performed on the $i$ th and $j$ th qudits. One important fact to note is that both of these gates are in the Clifford group. Since we have already constructed the Clifford group, this will enable us to also construct the Toffoli gate. The appropriate state is

$$
|A\rangle=\sum_{a, b}|a\rangle|b\rangle|a b\rangle
$$

Now, given three data qudits, perform inverse SUM gates (i.e., $|a\rangle|b\rangle \rightarrow$ $|a\rangle|b-a\rangle)$ from the first and second ancilla qudits to the first and second data qudits, respectively, and a SUM gate from the third data qudit to the third ancilla qudit. Now we measure the last three qudits, the original data qudits, in the bases $Z, Z$, and $X$, respectively. After performing the appropriate corrections (which will likely involve gates from the Clifford group as well as the Pauli group), we are left with the data in the first three qudits, which were originally the ancilla qudits. It turns out that after these operations, a Toffoli gate has been performed on the data qudits.

To construct the appropriate ancilla state $|A\rangle$, we can again follow Shor. The various states

$$
\left|A_{j}\right\rangle=\sum_{a, b}|a\rangle|b\rangle|a b+j\rangle
$$

for $j=0, \ldots, d-1$ are related to $|A\rangle$ by

$$
\left|A_{j}\right\rangle=\left(I \otimes I \otimes X^{j}\right)|A\rangle .
$$

The states $\left|A_{j}\right\rangle$, like $|A\rangle$, are +1 eigenstates of $M_{1}$ and $M_{2}$, but $M_{3}\left|A_{j}\right\rangle=\omega^{j}\left|A_{j}\right\rangle$. 
Furthermore, note that

$$
\sum_{j=0}^{d-1}\left|A_{j}\right\rangle=\sum_{a, b, c}|a\rangle|b\rangle|c\rangle .
$$

This last state is easily constructed as the Fourier transform of $|000\rangle$. Then by measuring $M_{3}$ for this state, we can collapse the state into one of the states $\left|A_{j}\right\rangle$. By applying the operator $X^{-j}$, we get the state $|A\rangle$.

To measure $M_{3}$ fault-tolerantly for a quantum code, we prepare the CAT state $\sum_{j}|j j \cdots j\rangle$ (using the same number of qudits as in a block of the code). $M_{3}$ is in the Clifford group, so we can perform it by some sequence of tranversal operations and measurements. By conditioning the appropriate operations for the $i$ th qudit on the $i$ th qudit of the CAT state, we can conditionally perform $M_{3}$ on the code depending on the CAT state. Note that a conditional operation in the $d$-dimensional case means applying $M_{3}^{j}$ when the control qudit is in the state $|j\rangle$. Once we have done this, when the code is in an eigenstate of $M_{3}$ with eigenvalue $\omega^{s}$, the CAT state ends up in the state

$$
\left|C A T_{s}\right\rangle=\sum_{j} \omega^{j s}|j j \cdots j\rangle
$$

The various states $\left|C A T_{s}\right\rangle$ are orthogonal to each other, and so can be distinguished by an appropriate measurement. This therefore gives us a measurement of $M_{3}$, completing the construction of the Toffoli gate and step 6 of the proof.

Because everything we do is transversal, single qudit errors in the CAT state cannot become more than single qudit errors in any single block of the code. Naturally, after creating the CAT state we should verify it to make sure there are no correlated errors. In addition, a single qudit error in the CAT state could give us the wrong measurement result. Therefore, the measurement of $M_{3}$ should be repeated in order to sufficiently increase our confidence in the result.

\section{Acknowledgements}

I would like to thank Manny Knill and Raymond Laflamme for helpful discussions, and Michael Nielsen for suggesting the name "Pauli group." This paper is preprint LA-UR 98-270.

\section{References}

[1] P. Shor, Phys. Rev. A 52, 2493 (1995).

[2] A. M. Steane, Phys. Rev. Lett. 77, 793 (1996).

[3] D. Gottesman, Phys. Rev. A 54, 1862 (1996), quant-ph/9604038

[4] A. R. Calderbank, E. M. Rains, P. W. Shor, and N. J. A. Sloane, Phys. Rev. Lett. 78, 405 (1997), quant-ph/9605005. 
[5] A. R. Calderbank, E. M. Rains, P. W. Shor, and N. J. A. Sloane, "Quantum error correction via codes over GF(4)," quant-ph/9608006, to appear in IEEE Trans. Information Theory.

[6] P. Shor, Proceedings of the 37th Symposium on the Foundations of Computer Science, IEEE Computer Society Press (Los Alamitos, CA), 56 (1996), quant$\mathrm{ph} / 9605011$.

[7] D. Gottesman, Phys. Rev. A 57, 127 (1998), quant-ph/9607029.

[8] E. Knill, "Non-binary error bases and quantum codes," quant-ph/9608048.

[9] E. Knill, "Group representations, error bases and quantum codes," quant$\mathrm{ph} / 9608049$.

[10] E. Rains, "Nonbinary quantum codes," quant-ph/9703048.

[11] D. DiVincenzo and P. Shor, Phys. Rev. Lett. 77, 3260 (1996), quant-ph/9605031.

[12] E. Knill and R. Laflamme, private communication.

This article was processed using the $\mathrm{LAT}_{\mathrm{E}} \mathrm{X}$ macro package with LLNCS style 